\title{
Dissecting Colloidal Stabilization Factors in Crowded Polymer Solutions by Forming Self-Assembled Monolayers on Gold Nanoparticles
}

\author{
Nathan J. Lang ${ }^{\dagger}$, Biwu Liu ${ }^{\dagger}$, Xu Zhang ${ }^{\dagger, \star}$ and Juewen Liu ${ }^{\dagger, *}$ \\ † Department of Chemistry and Waterloo Institute for Nanotechnology, ‡ Department of Biology, \\ University of Waterloo, 200 University Avenue West, Waterloo, Ontario, Canada N2L 3G1.
}

To whom correspondence may be addressed: liujw@uwaterloo.ca

\begin{abstract}
An ideal colloidal system should be highly stable in a diverse range of buffer conditions while still retaining its surface accessibility. We recently reported that dispersing citrate-capped gold nanoparticles (AuNPs) in polymers such as polyethylene glycol (PEG) can achieve such a goal due to contributions from depletion stabilization. Since AuNPs can weakly adsorb PEG to exert steric stabilization and the remaining citrate can impart charge stabilization, the extent of the contribution of depletion stabilization is unclear. In this work, we aim to dissect the contribution of each stabilizing factor. This is achieved by coating AuNPs with a layer of thiolated compound, which inhibits the adsorption of PEG and also allows the control of surface charge. We found that depletion stabilization alone was insufficient to stabilize AuNPs at room temperature. However, when working together with other stabilization mechanisms, ultrahigh stability can be achieved. The size of both AuNPs and PEG was systematically varied and the trend was compared with theoretical calculations. Finally, we report the importance of the surface chemistry of commercial AuNPs.
\end{abstract}

This document is the Accepted Manuscript version of a Published Work that appeared in final form in Langmuir, copyright $\odot$ American Chemical Society after peer review and technical editing by publisher. To access the final edited and published work see [insert ACS Articles on Request author-directed link to Published Work, see Lang, N. J., Liu, B., Zhang, X., \& Liu, J. (2013). Dissecting Colloidal Stabilization Factors in Crowded Polymer Solutions by Forming Self-Assembled Monolayers on Gold Nanoparticles. Langmuir, 29(20), 6018-6024. https://doi.org/10.1021/la3051093 


\section{Introduction}

An ideal colloid needs to withstand a diverse range of solution conditions including high salt, extreme $\mathrm{pH}$ and other chemicals that may adsorb onto the particle surface. ${ }^{1,2}$ Gold nanoparticles (AuNPs) are a very important class of colloidal material for analytical, catalytic and biomedical applications. ${ }^{3-9}$ Dispersed AuNPs display a vivid red color, whereas AuNP aggregation is accompanied by a color change to blue due to surface plasmon coupling. Such color change also makes AuNPs attractive for studying fundamental intermolecular forces that may affect colloidal stability.

Three mechanisms are commonly employed to stabilize nanoparticles. For AuNPs prepared using citrate as a reducing agent, citrate ions are believed to be adsorbed by the AuNPs to render a negatively charged surface allowing charge stabilization. While charge stabilization is easy to realize, it is sensitive to salt. Upon addition of just $30 \mathrm{mM} \mathrm{NaCl}$, for example, AuNPs start to aggregate. The second mechanism is the so-called depletion stabilization, where AuNPs are dispersed in a polymer solution. A polymer chain loses its configuration entropy when it is nearby a non-adsorbing surface. To avoid this, the polymer tends to stay away from the surface, creating a depletion zone (Figure 1A, the dashed ring). The width of the depletion zone is equal to the radius of the polymer molecule. This depletion effect makes the system crowded. If the depletion zone of two particles overlaps, the osmotic pressure from the surrounding polymers exerts an attractive force, which is known as depletion attraction. ${ }^{10-16}$ Depletion attraction is a thermodynamically favourable situation since more free volumes are created to relieve the crowdedness. Before depletion attraction can take place, however, there might be a repulsive barrier, which increases the colloidal stability and is called depletion stabilization or repulsion. The origin of depletion repulsion is related to the transportation of the polymers trapped between the particles against a deep osmotic pressure gradient (Figure 1B). ${ }^{16-18}$ The third mechanism is steric stabilization, and it occurs due to adsorbed polymers. ${ }^{19-22}$ For example, although polyethylene glycol (PEG) is generally considered to be a non-adsorbing polymer, a gold surface can still weakly adsorb PEG, which acts as a steric stabilizer for AuNPs to prevent them from directly touching each other. 
We recently reported that with a few percent of high MW PEG, citrate-capped AuNPs are stable even under extreme conditions such as with very high salt (e.g. > $1 \mathrm{M} \mathrm{Mg}^{2+}$ ) and extreme $\mathrm{pH}$ values (e.g. $\mathrm{pH} 1-13){ }^{2}$ At the same time, the surface accessibility of AuNPs is not hindered. Therefore, PEG provides a useful means to manipulate AuNPs. Based on the above discussions, although depletion stabilization plays an important role in this case, contributions from electrostatic (e.g. by the remaining surface citrate) and steric stabilization (e.g. by adsorbed PEG) cannot be completely ruled out (Figure 1D). The aim of this work is to understand the effect of each stabilizing factor, which was achieved by modifying the surface chemistry of AuNPs. For example, AuNPs can easily adsorbing thiolated compounds to form self-assembled monolayers (SAMs), ${ }^{23-26}$ which may inhibit the adsorption of PEG. The electrostatic interaction can be controlled by changing the tail group of the thiol compounds. Therefore, the SAM technology allows the elimination of one or two stabilizing mechanisms out of the three. We demonstrate that for AuNPs dispersed in PEG, all the three stabilization forces are needed to achieve high colloidal stability, suggesting that the contribution of each factor is comparable.
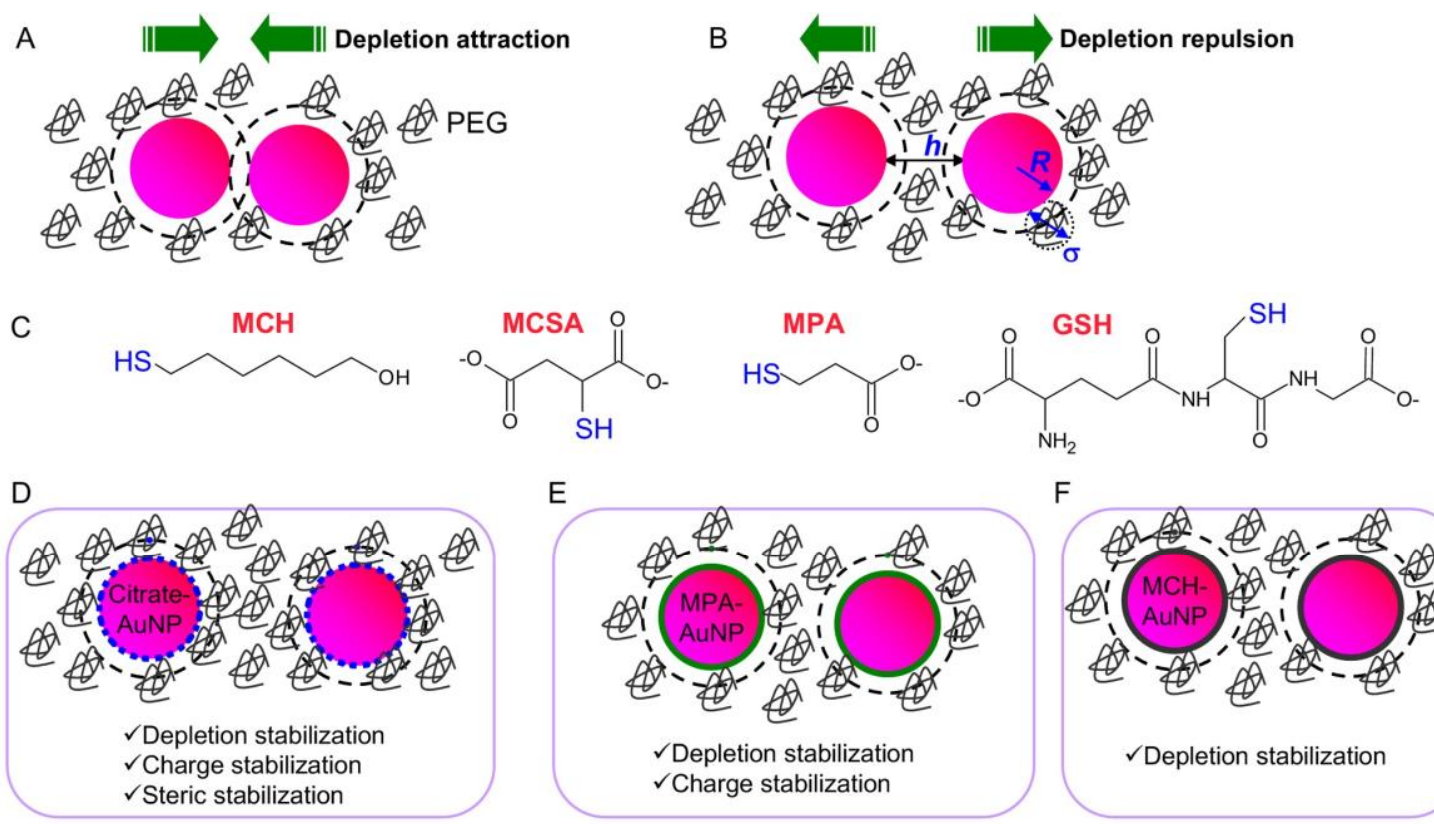

$\mathrm{F}$

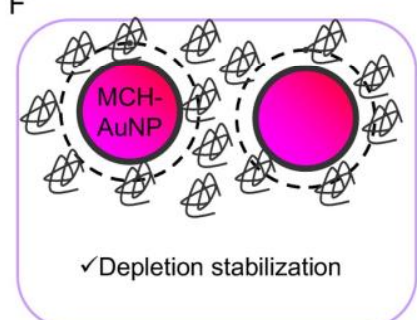

Figure 1. Schematics of attractive depletion force (A) when the depletion zones of two particles overlap and depletion repulsion (B), where the transportation of PEG between two particles is difficult. The inter- 
AuNP distance, AuNP radius and PEG diameter are denoted as $h, R$, and $\square$, respectively. (C) Structures of the thiol compounds used in this study. (D) Since PEG can be adsorbed onto citratecapped AuNPs, all the three stabilization mechanisms take place to stabilize AuNPs. Only a small amount of PEG is adsorbed by AuNPs while most other PEGs serve as a depletion agent. (E) Capping AuNPs by negatively charged MPA (3-mercaptopropionic acid), MCSA (mercaptosuccinic acid) or GSH (L-glutathione) eliminates steric stabilization since PEG cannot adsorb on these monolayers but the other two stabilization mechanisms are still effective. (F) Capping AuNPs with non-charged MCH (6mercapto-1-hexanol) leaves only depletion stabilization.

\section{Results and Discussion}

PEG adsorption on SAM-capped AuNPs. In this work, we employed a number of thiol compounds to displace citrate ions on AuNPs (see Figure 1C for structures). This allows a precise control of the surface charge and adsorption property of AuNPs. For example, it is easy to conceive that AuNPs become charge neutral upon adsorbing a monolayer of $\mathrm{MCH}$ (Figure 1F), while carboxyl containing compounds such as MPA, MCSA and GSH produce a negatively charged surface (Figure 1E). We chose these three negatively charged compounds because they have different sizes and contain different numbers of carboxyl groups.

A number of previous reports have shown that PEG can be adsorbed onto gold surface via the ether oxygen. ${ }^{2,18,27,28}$ Since PEG is a polymer, its adsorption onto AuNPs may provide steric stabilization. We reason that the adsorption affinity for PEG might be significantly reduced by forming SAMs on AuNPs. To test this hypothesis, FAM-labeled PEG 10k (e.g. MW=10,000) was mixed with AuNPs and various concentrations of $\mathrm{MCH}$, so that PEG adsorption could be monitored by decay of fluorescence. As shown in Figure 2A, the amount of fluorescence quenching was significantly reduced with increasing concentrations of $\mathrm{MCH}$. Therefore, $\mathrm{MCH}$ effectively lowered the affinity between PEG 
and AuNPs. To test the generality of this observation, we incubated the FAM-labeled PEG with AuNPs in the presence of various concentrations of the thiol compounds. After removing the free PEG using centrifugation, the AuNPs were dissolved with $\mathrm{KCN}$. The fluorescence of the samples was then measured to compare the amount of PEG remaining on the AuNPs (Figure 2B). Adsorbed PEG was found to drop significantly in the presence of all tested thiol compounds in a concentration-dependent manner. By using $100 \mathrm{DM}$ of any of the thiol compounds, PEG adsorption was completely eliminated. The concept of depletion zone is derived with non-adsorbing polymers (e.g. Figure 1A, B). In the AuNP-PEG system, however, PEG can be effectively adsorbed by AuNPs. We reason that the more precise model of this system is that AuNPs are covered by a layer of PEG and the rest of the PEG molecules in solution are repelled by the adsorbed PEG (Figure 2D). To test this hypothesis, we measured the hydrodynamic diameter of $13 \mathrm{~nm}$ AuNPs to be $15.5 \mathrm{~nm}$ (Figure 2C, dashed trace). After adding 1 पDM PEG (AuNP = $2 \mathrm{nM}$ ), the size went up only to $17.7 \mathrm{~nm}$. Further increase of PEG concentration to 62 पDM did not significantly change the measured size (inset of Figure 2C). In other words, the adsorbed PEG contributes to only $\sim 1 \mathrm{~nm}$ in the thickness of the hydrodynamic size of AuNPs and as such, PEG must be adsorbed flat on the AuNP surface as shown in Figure 2D. Otherwise, we expect a much larger particle size since the hydrodynamic size of PEG $20 \mathrm{k}$ is $\sim 10 \mathrm{~nm}$. This model also explains why adsorbed PEG is not a good steric stabilizer for AuNPs since it is not extended into solution but follows the contour of the particle surface. As a result, AuNPs can still approach to each other in very close proximity and experience a strong van der Waals attraction to aggregate. 

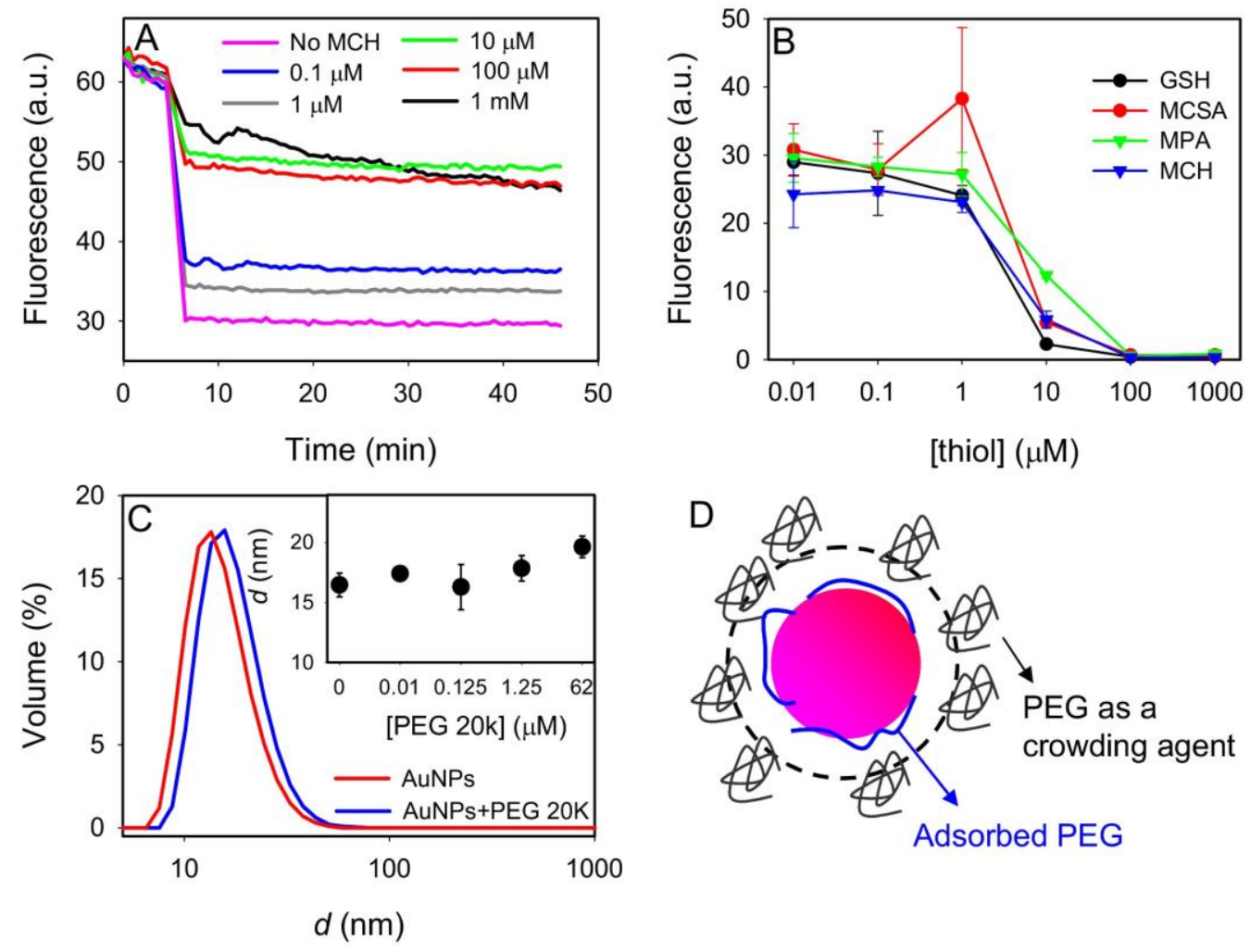

Figure 2. (A) Kinetics of FAM-labeled PEG 10k adsorption onto $13 \mathrm{~nm}$ AuNPs incubated with various concentrations of MCH. (B) Fluorescence of PEG-labeled PEG remaining on AuNP surface after incubating AuNPs with various concentrations of the thiol compounds. (C) AuNP (13 nm, $2 \mathrm{nM}$ ) size distribution measured with dynamic light scattering (DLS) in the absence or presence of 1 पM PEG 20k. Inset: change of size as a function of PEG 20k concentration. (D) Schematic of PEG interacting with AuNPs. Some PEGs are adsorbed by AuNPs (blue lines) and the rest of the PEG molecules act as depletion agents that are repelled by the PEG coated AuNPs. This is a more accurate representation than those shown in Figure 1.

Effect of SAM on AuNP aggregation kinetics. The above experiments confirmed the feasibility of using thiolated compounds to control the adsorption properties of AuNPs. Next, we study the stability of AuNPs as a function of its surface chemistry. We first added the thiol compounds to citrate-capped AuNPs without any PEG. An immediate color change to blue was observed only in the presence of 
MCH since it eliminated charge stabilization, the only stabilization mechanism available for these AuNPs (Figure 3A). All the other samples remained stable even after a full day since their surfaces were still negatively charged. The kinetics of color change was also followed using UV-vis spectroscopy. Dispersed $13 \mathrm{~nm}$ AuNPs have a sharp surface plasmon peak at $520 \mathrm{~nm}$ (Figure 3B, time $=0$ ). Upon addition of $\mathrm{MCH}$, the surface plasmon peak shifts to $\sim 650 \mathrm{~nm}$. Therefore, we used the extinction ratio of $650 \mathrm{~nm}$ over $520 \mathrm{~nm}$ to quantify the color of $13 \mathrm{~nm}$ AuNPs, where a high ratio indicates blue colored aggregates. We plotted the kinetics of AuNP color change by adding the different thiol compounds (Figure 3C); only $\mathrm{MCH}$ induced a fast color change.

Next, citrate-capped AuNPs were dispersed in 4\% PEG 20k and the color of the samples were monitored upon adding the thiol compounds. With $\mathrm{MCH}$, both electrostatic and steric stabilization were removed and only depletion stabilization remained (Figure 1F). As shown in Figure 3D, MCH still induced quick aggregation of AuNPs even in the presence of PEG. Therefore, depletion stabilization by 4\% PEG 20k alone was insufficient to stabilize MCH-capped AuNP. The other samples with carboxyl thiols did not change color, which is expected since the AuNPs were protected by both charge and depletion stabilization (Figure 1E). Therefore, depletion stabilization needs to act together with charge stabilization to protect these AuNPs.

The samples were then also challenged with $1 \mathrm{M} \mathrm{NaCl}$ to screen the charge stabilization (Figure 3E). In this case, all the samples showed color change with the aggregation rate being MCH $\square$ MPA $\square$ MCSA > GSH. To better distinguish between the three carboxyl compounds, we further raised $\mathrm{pH}$ to 11 to ensure that they were effectively deprotonated. In this case, MPA still induced a similarly fast aggregation rate as $\mathrm{MCH}$ (Figure 3F). On the other hand, the aggregation rate of MCSA was significantly reduced while GSH-capped AuNPs were fully stable. MPA has only a single carboxyl group, and it shows poor protection when challenged with $1 \mathrm{M} \mathrm{NaCl}$. It is likely that the negative charge was screened by the salt. MCSA is slightly larger with two carboxyl groups. In this case, we observed better protection, especially at higher $\mathrm{pH}$. GSH has the largest size among the three and it also has two carboxyl groups. 
Its strong stabilization effect suggests the importance of the size and the number of charges of the capping agents.

A
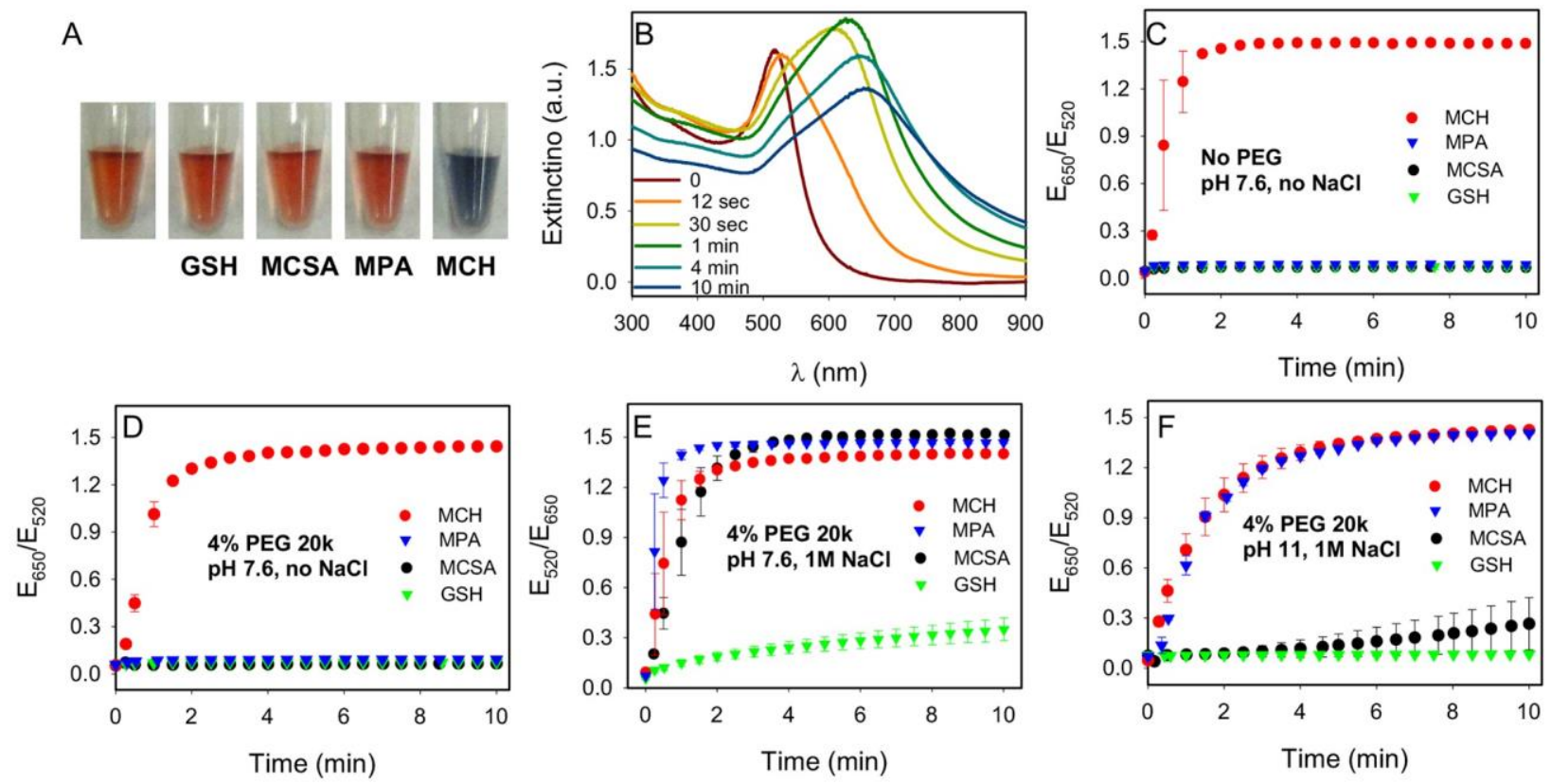

Figure 3. (A) A photograph of citrate-capped $13 \mathrm{~nm}$ AuNPs added with a final of $100 \mathrm{CM}$ of various thiol compounds at $\mathrm{pH} 7.6$ (5 mM HEPES); no $\mathrm{NaCl}$ or PEG was present. (B) Evolution of UV-vis spectra of $13 \mathrm{~nm}$ AuNPs after adding $100 \mathrm{CM}$ MCH. No PEG was present in this sample. (C-F) Kinetics of the AuNP extinction ratio change in the presence of $100 \mathrm{CM}$ of the four thiol compounds in different buffer conditions. (C): pH 7.6, no NaCl, no PEG; (D): pH 7.6, no NaCl with 4\% PEG 20k; (E): pH 7.6, with $1 \mathrm{M} \mathrm{NaCl}$ and 4\% PEG 20k; (F): pH 11, with $1 \mathrm{M} \mathrm{NaCl}$ and 4\% PEG 20k.

Effect of AuNP size and PEG MW. One of the advantages of using AuNPs is that their size can be precisely controlled. Many size-dependent studies have been carried out using AuNPs including optical properties, ${ }^{29}$ melting properties, ${ }^{30}$ DNA density, ${ }^{31}$ macromolecular crowding effect, ${ }^{32}$ cellular uptake and toxicity. ${ }^{33}$ To test the depletion force, we employed AuNPs from $13 \mathrm{~nm}$ to $100 \mathrm{~nm}$ and they were 
respectively dispersed in $10 \%$ PEG 20k. Aggregation was initiated by adding $\mathrm{MCH}$, which eliminates both charge and steric stabilization. As shown in Figure 4, significant spectral shift is observed for all of the AuNP sizes and the amount of change appears to be less significant for larger AuNPs.
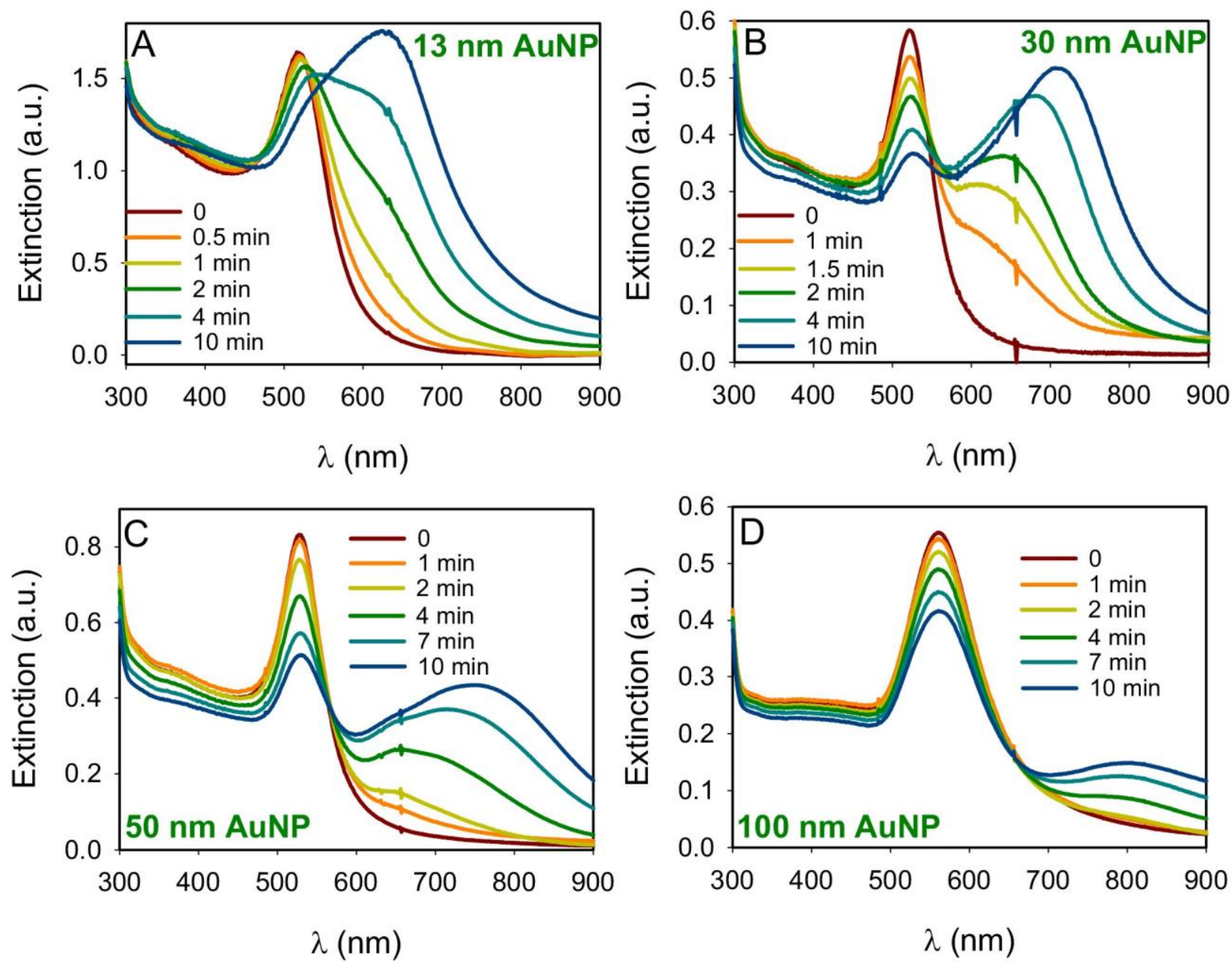

Figure 4. UV-vis spectra as a function of time for AuNPs of different sizes dispersed in 10\% PEG 20k after addition of $100 \mathrm{DM} \mathrm{MCH}$. AuNP size $=13 \mathrm{~nm}(\mathrm{~A}), 30 \mathrm{~nm}(\mathrm{~B}), 50 \mathrm{~nm}(\mathrm{C})$, and $100 \mathrm{~nm}$ (D).

To have a further understanding on whether the MW of PEG also plays a role, the AuNPs were dispersed in 10\% PEG of various MWs. Since the plasmon peak and its shift upon aggregation were 
different for AuNPs of different size, different wavelength ratios were chosen for each particle. After the first measurement, a final concentration of $100 \mathrm{DM}$ of $\mathrm{MCH}$ was added to initiate aggregation. Again, under this condition, AuNPs were protected only by depletion stabilization. As shown in Figure 5A, 10\% PEG 2k and 4k did not appear to stabilize $13 \mathrm{~nm}$ AuNPs and the AuNPs even aggregated slightly faster than those dispersed in water. PEG 8k and PEG 20k retarded aggregation but no complete protection was achieved. For the three larger AuNPs, all the PEGs showed protection and the overall trend was that larger PEG sizes were more effective. It is interesting to note that AuNPs aggregated faster in PEG 4k than in PEG 2k though (PEG 5B-D).

Theoretical calculations point out the depletion repulsion barrier $W_{d}=\left[R \square^{2} / 5 \square\right] f(h)$, where $R$ is the radius of AuNP, $\square$ is the diameter of PEG, $\square$ is the volume fraction of PEG, and $f(h)$ is a function of distance $h$ between two AuNPs (see Figure 1B for the these definitions). ${ }^{15,16}$ A plot of the potential energy caused by depletion interactions is shown in Figure 5E, where the repulsive barrier height is reached when the inter-particle distance $h=\square(1-3 \square / 2)$. At this distance, the barrier height is $12 R \square^{2} / 5 \square$, with the unit being $k T$ (the thermal energy). We plotted the barrier height for $10 \%$ PEG 2k, 4k and 8k in Figure 5F. Note that PEG 20k reaches the semi-dilute concentration at 5.1\% (w/w) concentration, ${ }^{34}$ where the hard sphere model breaks down. PEG $8 \mathrm{k}$ reaches the semi-dilute concentration at $\sim 10 \%$ and its calculation is unlikely to be accurate either since the factor of $1-3 \square / 2$ is negative when the volume fraction $\square$ is $\sim 1$. Nevertheless, the equation indeed predicts that PEG $2 \mathrm{k}$ has a higher barrier height than PEG 4k, consistent with our experimental results. We further compared the aggregation of $100 \mathrm{~nm}$ AuNPs in 2 mM PEG of various MWs, where higher MW PEGs showed better protection, which is also expected for depletion protection (Figure S1, Supporting Information). Note that the above calculations were based on weight percentage. 
If the PEG size and concentration is fixed, the depletion repulsion barrier should scale linearly with the size of AuNPs as shown by the above equation. This is supported by our data that $13 \mathrm{~nm}$ AuNPs aggregated faster in PEG 2k or 4k (Figure 5A) but the larger AuNPs were slightly protected by these small PEGs. It is difficult to directly compare the aggregation rate of different AuNPs since we monitored only the extinction ratio and the relationship between this ratio and aggregation is not straightforward. Overall, by just judging from this ratio change, depletion stabilization is only moderately dependent on the size of AuNPs in the range of 13 to $100 \mathrm{~nm}$.
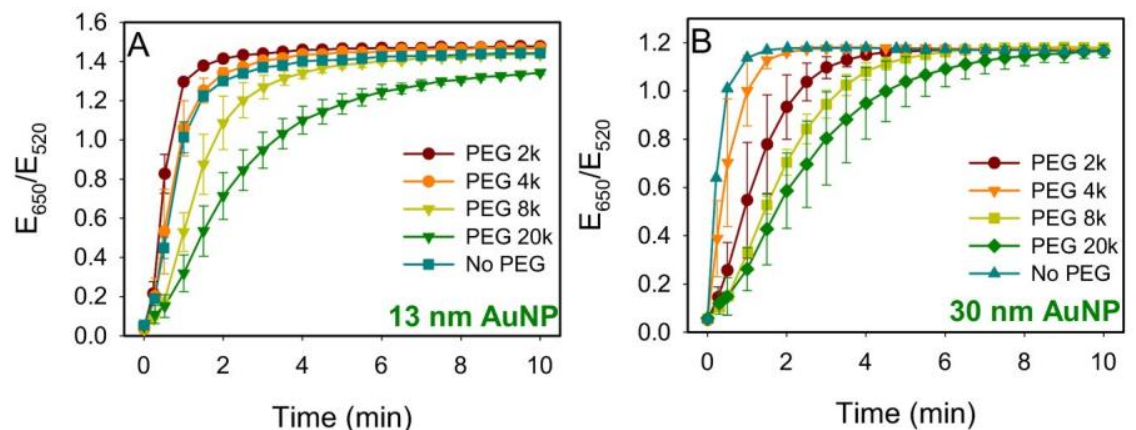

$E$
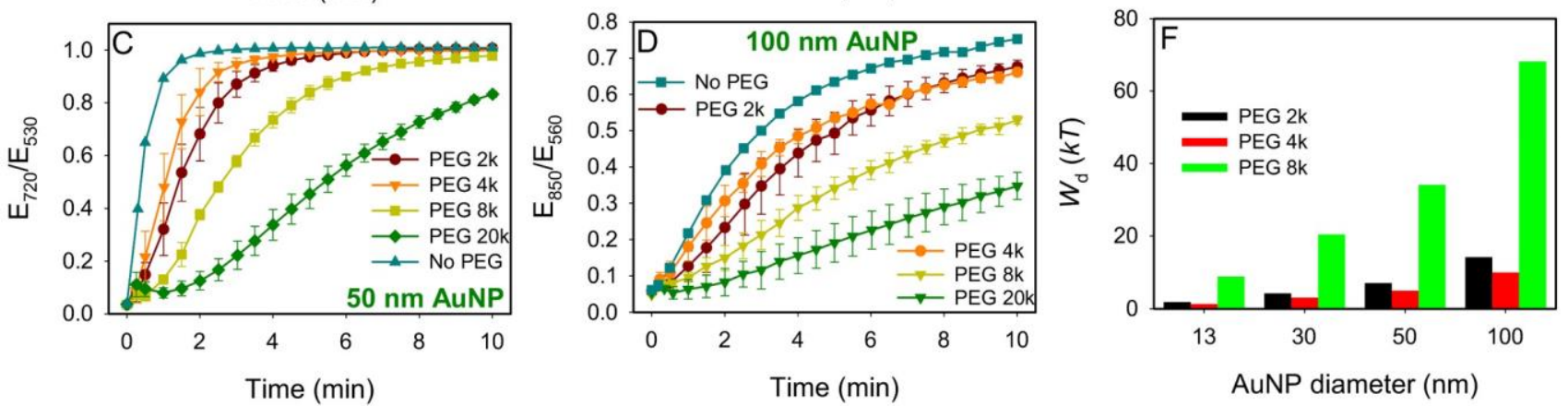

Figure 5. Kinetics of AuNP aggregation upon addition of $\mathrm{MCH}$ as a function of PEG MW for $13 \mathrm{~nm}$ (A), $30 \mathrm{~nm}(\mathrm{~B}), 50 \mathrm{~nm}(\mathrm{C})$, and $100 \mathrm{~nm}$ (D) AuNPs. (E) Depletion potential between two AuNPs mediated by PEG modeled as hard spheres. Drawing not to scale. (F) Relative depletion repulsion barrier height as a function of AuNP size and PEG size.

The change of AuNP extinction is a strong function of the size AuNPs. For example, very little change is expected when two $13 \mathrm{~nm}$ AuNPs form a dimer but quite large change might be produced with the 
formation of $100 \mathrm{~nm}$ dimers. To further understand the aggregation of AuNPs in PEG, we monitored the growth of particle size upon addition of MCH using DLS. For 13 nm AuNPs dispersed in 4\% PEG 2k, an immediate increase in size to greater than $200 \mathrm{~nm}$ was observed just in $10 \mathrm{sec}$ after adding $\mathrm{MCH}$ (Figure 6A). After $10 \mathrm{~min}$, the average size went up to $\sim 1 \mathrm{Dm}$. The kinetic trace in Figure $6 \mathrm{~B}$ also reflects this trend (Figure 6B, blue dots). Therefore, for $13 \mathrm{~nm}$ AuNPs, large aggregates containing tens to hundreds of nanoparticles were produced. On the other hand, the size change for $100 \mathrm{~nm}$ AuNPs was quite moderate to only $\sim 130 \mathrm{~nm}$ (Figure 6B, red dots). Therefore, it is likely that $100 \mathrm{~nm}$ AuNPs just formed dimers. This experiment further suggests that larger AuNPs were much more effectively protected. It needs to be pointed out that the concentration of large AuNPs was significantly lower compared to the smaller ones, which may also contribute to the observed slower aggregation. While our UV-vis data reflected this difference that $13 \mathrm{~nm}$ AuNPs showed a faster change in the extinction ratio, the DLS data provided more quantitative information about the nanoscale picture. Therefore, caution needs to be taken when interpreting the UV-vis data, where it is not straightforward to correlate the spectral shift to the aggregation states. Comparison can only be made for the same sized AuNPs.
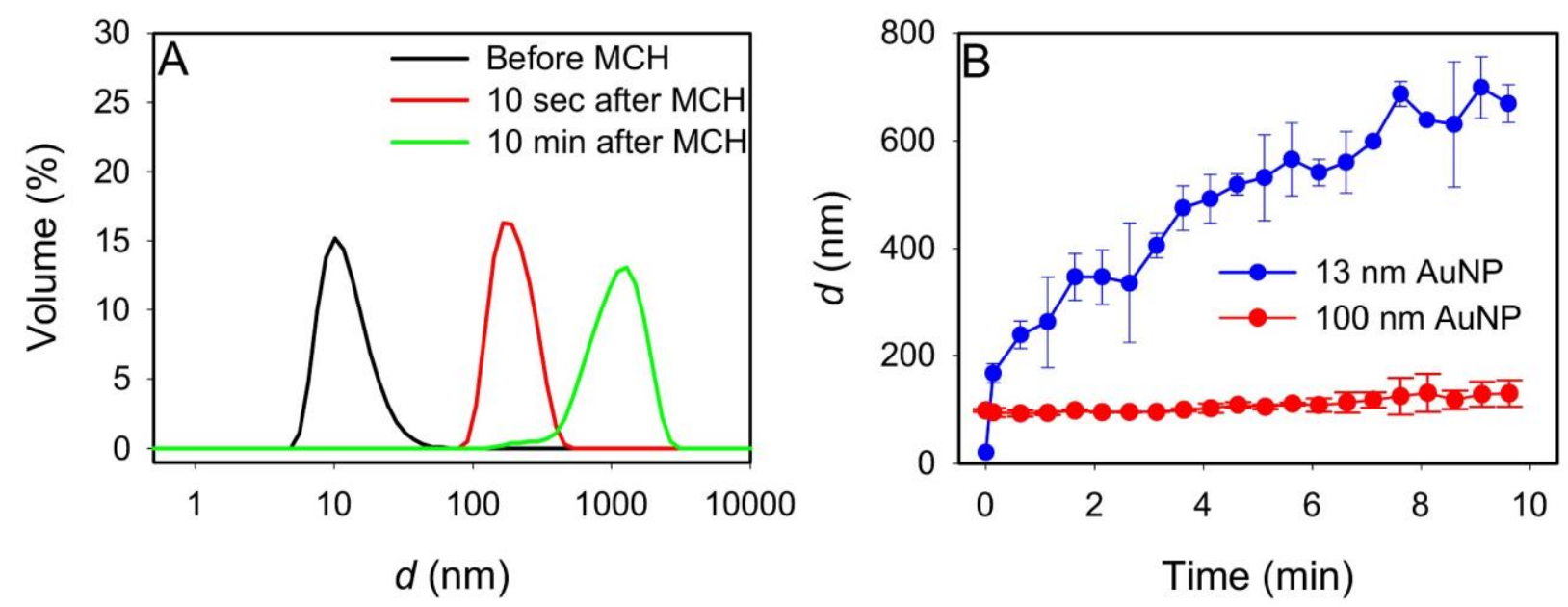

Figure 6. (A) DLS spectra of $13 \mathrm{~nm}$ AuNPs dispersed in 4\% PEG $2 \mathrm{k}$ before and after adding $0.1 \mathrm{mM}$ MCH. (B) Kinetics of change of the average size of 13 and $100 \mathrm{~nm}$ AuNPs after adding MCH. 
While high quality AuNPs smaller than $20 \mathrm{~nm}$ can be produced using citrate reduction in many labs, most researchers obtain larger AuNPs from commercial sources. We initially used 20-100 AuNPs from BBInternational, where the surface chemistry of the AuNPs was not disclosed to us. We found that the aggregation of $30 \mathrm{~nm}$ AuNPs was very slow and 50 and $100 \mathrm{~nm}$ AuNPs were completely protected from $\mathrm{MCH}$ even in the absence of PEG (see Figure S2, S3, Supporting Information). Then we purchased the same sized AuNPs from nanoComposix Inc., where the surface of AuNPs was confirmed to be capped by citrate. In this case, $\mathrm{MCH}$ induced aggregation was observed and the data in

Figure 4, 5 were collected using these citrate-capped AuNPs. This suggests that the large AuNPs from BBInternational might contain ligands that might not be completely displaced by $\mathrm{MCH}$, leading to other protection mechanisms in addition to depletion stabilization.

\section{Summary}

In this work, we controlled the surface property of AuNPs using the SAM technology. All the tested thiol compounds eliminated PEG adsorption and the surface charge was controlled by changing the tail groups. This allowed us to explore various forces responsible for stabilizing colloidal systems, in particular, the depletion repulsion force. The AuNP size was tested from 13 to $100 \mathrm{~nm}$, and the size of PEG was also systematically varied. ${ }^{35}$ Since the aggregation of AuNPs can be monitored by its color change, this is a useful system to understand fundamental colloidal sciences. Compared to the commonly used silica or latex particles for studying depletion force, our AuNPs are at a much lower volume fraction. For example, $10 \mathrm{nM} 13 \mathrm{~nm}$ AuNPs have a volume fraction of only $0.0007 \%$. The volume fractions of larger AuNPs are even smaller, while even $1 \%$ volume fraction of silica particles is considered to be low. With a high colloidal concentration, previous work has been focused on the phase diagram of the colloid/polymer systems, ${ }^{36-40}$ where the $x$-axis is usually the polymer concentration and $y$-axis is the particle concentration. When either or both components reach a high concentration, phase separation may occur. In our system, the nanoparticle concentration (volume fraction) is close to zero. 
Without added salt, high MW PEGs alone do not have adverse effect on the colloidal stability of AuNPs. Therefore, AuNPs provide a convenient tool to study low volume fraction particles dispersed in depletion agents. From this study, we conclude that the ultrahigh stability of AuNPs dispersed in PEG was achieved by an additive effect of charge, steric and depletion stabilization.

\section{Materials and Methods}

Chemicals. AuNPs of $13 \mathrm{~nm}$ diameter were prepared by the standard citrate reduction method. ${ }^{41}$ Other sized AuNPs were purchased either from BBInternational through Ted Pella Inc. (Redding, CA), where the surface chemistry is unclear or from nanoComposix Inc (San Diego, CA), where AuNPs are capped by citrate. The size variation of the AuNPs was within 20\%. All the thiolated compounds were purchased from Sigma-Aldrich. NaCl, 4-(2-hydroxyethyl)-1-piperazineethanesulfonic acid (HEPES), and trisodium citrate were purchased from Mandel Scientific (Guelph, Ontario, Canada). The PEGs were purchased from VWR and the FAM-labeled PEG was from NanoCS. The thiol compounds were prepared fresh each week by dissolving them in water. The stock solutions were stored in -20 पC. The diluted solutions required for the tests were prepared fresh each day.

PEG adsorption kinetics. To monitor PEG adsorption kinetics as a function of AuNP surface modification, AuNPs (10 nM, $13 \mathrm{~nm}$ ) were respectively mixed with $1 \mathrm{mM}, 100 \mathrm{CM}, 10 \mathrm{MM}, 1 \mathrm{\square M}, 100$ $\mathrm{nM}$, and 0 of $\mathrm{MCH}$. In a 96-well plate, 89 पL of $5 \mathrm{mM}$ HEPES (pH 7.6) was mixed with $1 \mathrm{nM}$ FAMlabeled PEG 10k in six different wells. After monitoring the initial fluorescence for 5 min using a microplate reader (Infinite 200F, Tecan), $10 \mathrm{LL}$ from the $\mathrm{MCH}$ treated AuNP samples were added and fluorescence intensity was monitored for another $40 \mathrm{~min}$. The hydrodynamic sizes of AuNPs and PEG 20k adsorbed AuNPs were measured by dynamic light scattering (Zetasizer Nano, Malvern) at 25 पC.

PEG adsorption capacity. AuNPs (10 nM, 13 nm) were respectively mixed with $1 \mathrm{mM}, 100 \mathrm{\square M}, 10$ 
$\square \mathrm{M}, 1 \mathrm{MM}, 100 \mathrm{nM}$, and 0 of the thiolated compounds (GSH, MCSA, MPA, or MCH). After overnight incubation, approximately a final concentration of 1.4 DM FAM-PEG 10k was added to each sample and the samples were incubated for $40 \mathrm{~min}$. The samples were then centrifuged for $8 \mathrm{~min}$ at 15,000 rpm and rinsed with milli-Q water for four times. The remaining AuNP precipitants were dissolved with $\mathrm{KCN}$ to release adsorbed PEG. 10 LL of the dissolved samples were mixed with 90 CL of 5 mM HEPES 7.6 in a 96-well plate to read its fluorescence. The experiment was run in triplicate.

AuNP aggregation kinetics. The aggregation of AuNPs was monitored using a UV-vis spectrometer (Agilent 8453A). A 100 LL solution was used for each test, with a $13 \mathrm{~nm}$ AuNP concentration of $5 \mathrm{nM}$. AuNPs of other sizes were used by diluting the received materials by half. The molar concentrations of the stock solution of AuNPs are $10 \mathrm{nM}(13 \mathrm{~nm}), 1.2 \mathrm{nM}(20 \mathrm{~nm}), 0.3 \mathrm{nM}(30 \mathrm{~nm}), 0.075 \mathrm{nM}(50 \mathrm{~nm})$ and $0.0093 \mathrm{nM}(100 \mathrm{~nm})$. After an initial scan, one microliter of thiolated compound was added and the sample was monitored every $30 \mathrm{sec}$ for $10 \mathrm{~min}$. The cuvette was gently agitated before each reading to ensure good mixing. To test the effect of different thiol compounds, 4\% PEG was used for the experiments. To study the effect of adding salt, a final concentration of $0.9 \mathrm{M} \mathrm{NaCl}$ was included in each sample. For the $\mathrm{pH}$ test, the samples were adjusted to $\mathrm{pH} 11$ using $\mathrm{NaOH}$. To test different AuNP sizes, $10 \%$ PEG of various MWs were used for each sample and the final thiol compound concentration was $100 \mathrm{DM}$ for all the tests.

Dynamic light scattering. The size change of $13 \mathrm{~nm}$ and $100 \mathrm{~nm}$ AuNPs was monitored using DLS with a Malvern Instruments Zetasizer Nano-ZS90. Three conditions were tested for each particles size: no PEG, 4\% PEG 2k (20 mM), and 4\% PEG 20k (2 mM). After measuring the initial size and initial UV-vis absorbance of the particles, they were mixed with $100 \mu \mathrm{M} \mathrm{MCH}$ and monitored for ten minutes at 30 second intervals in the Zetasizer. Immediately after the last DLS measurement, the final UV-vis absorption was measured. This set of experiments was duplicated. For kinetic measurement, the sample was pre-incubated in the instrument to reach a stable temperature of $25 \square C$. Subsequent readings are 
adding $\mathrm{MCH}$ was performed without any waiting time. It needs to be noted that at high PEG concentration, the viscosity of the solutions increased drastically, which needs to be corrected to obtain correct size values. We used viscosity values of $1.2 \mathrm{cp}$ for $4 \%$ PEG $2 \mathrm{k}$ and $3.2 \mathrm{cp}$ for $4 \%$ PEG 20k.

\section{Acknowledgements}

Funding for this work is from the University of Waterloo, the Canadian Foundation for Innovation, and the Natural Sciences and Engineering Research Council (NSERC) of Canada and the Early Researcher Award from the Ontario Ministry of Research and Innovation.

Supporting Information Available. Aggregation of AuNPs from the other sources, aggregation of AuNPs as a function of PEG MW, calculations. This material is available free of charge via the Internet at http://pubs.acs.org

\section{References}

(1) Tohver, V.; Smay, J. E.; Braem, A.; Braun, P. V.; Lewis, J. A. Nanoparticle Halos: A New Colloid Stabilization Mechanism. Proc. Natl. Acad. Sci. U.S.A. 2001, 98, 8950-8954.

(2) Zhang, X.; Servos, M. R.; Liu, J. Ultrahigh Nanoparticle Stability against Salt, pH and Solvent with Retained Surface Accessibility via Depletion Stabilization. J. Am. Chem. Soc. 2012, 134, 99109913.

(3) Daniel, M.-C.; Astruc, D. Gold Nanoparticles: Assembly, Supramolecular Chemistry, Quantum-Size-Related Properties, and Applications toward Biology, Catalysis, and Nanotechnology. Chem. Rev. 2004, 104, 293-346.

(4) Saha, K.; Agasti, S. S.; Kim, C.; Li, X.; Rotello, V. M. Gold Nanoparticles in Chemical and Biological Sensing. Chem. Rev. 2012, 2739-2779.

(5) Giljohann, D. A.; Seferos, D. S.; Daniel, W. L.; Massich, M. D.; Patel, P. C.; Mirkin, C. A. 
Gold Nanoparticles for Biology and Medicine. Angew. Chem. Int. Ed. 2010, 49, 3280-3294.

(6) Rosi, N. L.; Mirkin, C. A. Nanostructures in Biodiagnostics. Chem. Rev. 2005, 105, 1547-1562.

(7) Zhao, W.; Brook, M. A.; Li, Y. Design of Gold Nanoparticle-Based Colorimetric Biosensing Assays. ChemBioChem 2008, 9, 2363-2371.

(8) Liu, J.; Cao, Z.; Lu, Y. Functional Nucleic Acid Sensors. Chem. Rev. 2009, 109, 1948-1998.

(9) Wang, H.; Yang, R. H.; Yang, L.; Tan, W. H. Nucleic Acid Conjugated Nanomaterials for Enhanced Molecular Recognition. ACS Nano 2009, 3, 2451-2460.

(10) Asakura, S.; Oosawa, F. Interaction between Particles Suspended in Solutions of Macromolecules. J. Polymer Sci. 1958, 33, 183-192.

(11) Asakura, S.; Oosawa, F. On Interaction between 2 Bodies Immersed in a Solution of Macromolecules. J. Chem. Phys. 1954, 22, 1255-1256.

(12) Joanny, J. F.; Leibler, L.; Degennes, P. G. Effects of Polymer-Solutions on Colloid Stability. J. Polymer Sci. B 1979, 17, 1073-1084.

(13) Vrij, A. Polymers at Interfaces and Interactions in Colloidal Dispersions. Pure Appl. Chem. 1976, $48,471-483$.

(14) Gast, A. P.; Hall, C. K.; Russel, W. B. Phase Separations Induced in Aqueous Colloidal Suspensions by Dissolved Polymer. Faraday Discuss. 1983, 76, 189-201.

(15) Lekkerkerker, H. N. W.; Tuinier, R., Colloids and the Depletion Interaction. New York, 2011.

(16) Mao, Y.; Cates, M. E.; Lekkerkerker, H. N. W. Depletion Force in Colloidal Systems. Physica A 1995, 222, 10-24.

(17) Semenov, A. N. Theory of Colloid Stabilization in Semidilute Polymer Solutions. Macromolecules 2008, 41, 2243-2249.

(18) Shin, J.; Zhang, X.; Liu, J. DNA-Functionalized Gold Nanoparticles in Macromolecularly Crowded Polymer Solutions. J. Phys. Chem. B 2012, 116, 13396-13402. 
(19) Dolan, A. K.; Edwards, S. F. Theory of Stabilization of Colloids by Adsorbed Polymer. Proceedings of the Royal Society of London Series A 1974, 337, 509-516.

(20) Brewer, S. H.; Glomm, W. R.; Johnson, M. C.; Knag, M. K.; Franzen, S. Probing BSA Binding to Citrate-Coated Gold Nanoparticles and Surfaces. Langmuir 2005, 21, 9303-9307.

(21) Hooper, J. B.; Schweizer, K. S. Contact Aggregation, Bridging, and Steric Stabilization in Dense Polymer-Particle Mixtures. Macromolecules 2005, 38, 8858-8869.

(22) Surve, M.; Pryamitsyn, V.; Ganesan, V. Nanoparticles in Solutions of Adsorbing Polymers: Pair Interactions, Percolation, and Phase Behavior. Langmuir 2006, 22, 969-981.

(23) Love, J. C.; Estroff, L. A.; Kriebel, J. K.; Nuzzo, R. G.; Whitesides, G. M. Self-Assembled Monolayers of Thiolates on Metals as a Form of Nanotechnology. Chem. Rev. 2005, 105, 11031169.

(24) Browne, K. P.; Grzybowski, B. A. Controlling the Properties of Self-Assembled Monolayers by Substrate Curvature. Langmuir 2011, 27, 1246-1250.

(25) Liu, X.; Huang, H.; Jin, Q.; Ji, J. Mixed Charged Zwitterionic Self-Assembled Monolayers as a Facile Way to Stabilize Large Gold Nanoparticles. Langmuir 2011, 27, 5242-5251.

(26) Gao, J.; Huang, X.; Liu, H.; Zan, F.; Ren, J. Colloidal Stability of Gold Nanoparticles Modified with Thiol Compounds: Bioconjugation and Application in Cancer Cell Imaging. Langmuir 2012, $28,4464-4471$.

(27) Mendez, A.; Moron, L. E.; Ortiz-Frade, L.; Meas, Y.; Ortega-Borges, R.; Trejo, G. Thermodynamic Studies of PEG (Mw 20,000) Adsorption onto a Polycrystalline Gold Electrode. J. Electrochem. Soc. 2011, 158, F45-F51.

(28) Zhang, X.; Huang, P.-J. J.; Servos, M. R.; Liu, J. Effects of Polyethylene Glycol on DNA Adsorption and Hybridization on Gold Nanoparticles and Graphene Oxide. Langmuir 2012, 28, 1433014337. 
(29) Kelly, K. L.; Coronado, E.; Zhao, L. L.; Schatz, G. C. The Optical Properties of Metal Nanoparticles: The Influence of Size, Shape, and Dielectric Environment. J. Phys. Chem. B 2003, $107,668-677$.

(30) Jin, R.; Wu, G.; Li, Z.; Mirkin, C. A.; Schatz, G. C. What Controls the Melting Properties of DNA-Linked Gold Nanoparticle Assemblies? J. Am. Chem. Soc. 2003, 125, 1643-1654.

(31) Hill, H. D.; Millstone, J. E.; Banholzer, M. J.; Mirkin, C. A. The Role Radius of Curvature Plays in Thiolated Oligonucleotide Loading on Gold Nanoparticles. ACS Nano 2009, 3, 418-424.

(32) Zaki, A.; Dave, N.; Liu, J. Amplifying the Macromolecular Crowding Effect Using Nanoparticles. J. Am. Chem. Soc. 2012, 134, 35-38.

(33) Chithrani, B. D.; Ghazani, A. A.; Chan, W. C. W. Determining the Size and Shape Dependence of Gold Nanoparticle Uptake into Mammalian Cells. Nano Lett. 2006, 6, 662-668.

(34) Ziebacz, N.; Wieczorek, S. A.; Kalwarczyk, T.; Fialkowski, M.; Holyst, R. Crossover Regime for the Diffusion of Nanoparticles in Polyethylene Glycol Solutions: Influence of the Depletion Layer. Soft Matter 2011, 7, 7181-7186.

(35) Kuga, S. Pore Size Distribution Analysis of Gel Substances by Size Exclusion Chromatography. J. Chromatogr. A 1981, 206, 449-461.

(36) Pusey, P. N.; van Megen, W. Phase Behaviour of Concentrated Suspensions of Nearly Hard Colloidal Spheres. Nature 1986, 320, 340-342.

(37) Poon, W. C. K. The Physics of a Model Colloid-Polymer Mixture. J. Phys. Condens. Mat. 2002, 14, R859-R880.

(38) Ilett, S. M.; Orrock, A.; Poon, W. C. K.; Pusey, P. N. Phase-Behavior of a Model ColloidPolymer Mixture. Phys. Rev. E 1995, 51, 1344-1352.

(39) Tuinier, R.; Smith, P. A.; Poon, W. C. K.; Egelhaaf, S. U.; Aarts, D.; Lekkerkerker, H. N. W.; Fleer, G. J. Phase Diagram for a Mixture of Colloids and Polymers with Equal Size. EPL 2008, 82. 
(40) Kim, S. Y.; Zukoski, C. F. Particle Restabilization in Silica/PEG/Ethanol Suspensions: How Strongly Do Polymers Need to Adsorb to Stabilize against Aggregation? Langmuir 2011, 27, 52115221.

(41) Storhoff, J. J.; Elghanian, R.; Mucic, R. C.; Mirkin, C. A.; Letsinger, R. L. One-Pot Colorimetric Differentiation of Polynucleotides with Single Base Imperfections Using Gold Nanoparticle Probes. J. Am. Chem. Soc. 1998, 120, 1959-1964. 